\title{
ON THE ACCURACY OF THE STATIONARY FORCED KORTEWEG-DE VRIES EQUATION AS A MODEL EQUATION FOR FLOWS OVER A BUMP
}

\author{
BY \\ SAMUEL SHAN-PU SHEN \\ University of Alberta, Canada
}

\begin{abstract}
Considered are the stationary supercritical free-surface solitary waves and subcritical free-surface hydraulic falls in flows over a bump in a two-dimensional channel. The stationary forced Korteweg-de Vries equation (sfKdV) sometimes can be used to predict these solitary waves and hydraulic falls. This paper studies the deviation of the results deduced from the sfKdV from the exact solutions found by computations and experiments. The elucidation of this deviation (called the error) is by comparing the sfKdV results with the computational and experimental results since an analytic approach seems impossible. From the comparisons made in this paper and the previous studies in [6], we conclude that the sfKdV is a good model in the sense that the relative error is less than $10 \%$ for small or moderate amplitude free-surface elevation or depression. This corresponds to the bumps whose height is less than one half of the upstream depth and whose base is not longer than twice its height. When the height of a bump is larger than one half of the upstream depth and the base of the bump is very long, the error due to the sf KdV approximation is generally very large. In this case, the applicable asymptotic theory is that due to Green-Naghdi. The novel points included in this paper are: a new derivation of the $s f K d V$, a proof of an existence theorem of the positive solutions to the $s f K d V$, and an error analysis by comparisons.
\end{abstract}

1. Introduction. Sixteen years ago, in an impressive survey on the Korteweg-de Vries equation (KdV), Miura listed seven open problems on the KdV [1]. The seventh open problem is on the forced Korteweg-de Vries equation (fKdV). At that time the physical basis for the fKdV was not clear. It seems that Patoine and Warn were the first who, as early as 1982 , used the $\mathrm{fKdV}$ as a physical model equation [2]. However, it was not until 1984 that Akylas first systematically derived the fKdV from the model of long nonlinear water waves forced by a moving pressure [3]. Since 1985, there have been many papers published on the fKdV. The fKdV equations were derived for long water waves in a two-dimensional channel forced by a bottom topography and/or an external pressure applied on the free surface (see [4]-[5] and the references therein).

Received March 30, 1993.

1991 Mathematics Subject Classification. Primary 76B15, 35Q53.

(C)1995 Brown University 
All these works inevitably raised a question: how accurate is the fKdV as a model equation for the forced flows? It is this question that the present paper addresses. We wish to find out the approximate range of physical parameters, in which the fKdV can be applied with some confidence. Needless to say, it is important to know this range from the viewpoints of both theoretical analysis and practical applications. We do not intend, it may be impossible, to address the error problem in the fKdV asymptotic analysis in the sense of absolute precision via mathematical proofs. We conclude that the $\mathrm{fKdV}$ is an accurate model equation when the amplitude of the forcing is less than one half of the upstream depth and the base of the forcing is not longer than twice the forcing amplitude.

In our formal asymptotic expansion, the forcing due to the bottom bump is assumed small (of order $\varepsilon^{2}$, where $\varepsilon>0$ is a small number). The elevation response of the free surface to the forcing is assumed to be of the first order (of order $\varepsilon$ ) and hence is one order larger than the forcing. When the upstream velocity of the uniform flow is near the critical speed of the shallow water waves, the first-order elevation of the free surface satisfies an $\mathrm{fKdV}$. This $\mathrm{fKdV}$ is then used as the mathematical model equation to describe the nonlinear surface waves in the current forced by the bottom bump. Let $F$ be the Froude number, which is the ratio of the upstream uniform velocity $c^{*}$ to the critical speed of the shallow water waves $\sqrt{g H}$ (i.e., $F=c^{*} / \sqrt{g H}$ ). From the fKdV model, computations and experiments, it has been found that there exist two important values of the Froude number, $F_{C}$ and $F_{L}$, such that:

1. Supercritical solitary waves: When $F \geq F_{C}$, there exist at least two stationary solitary waves sustained on the bump. When $F \rightarrow F_{C}+0$, the two solitary waves merge into one.

2. Subcritical cnoidal waves and hydraulic fall: When $F<F_{L}$, the steady-state free surface consists of a downstream cnoidal wave matched with an upstream null solution. When $F \rightarrow F_{L}-0$, the period of the downstream cnoidal wave approaches infinity and the free surface becomes a hydraulic fall.

3. Transcritical upstream soliton radiation: For some $F$ in $\left(F_{L}, F_{C}\right)$, the flow never approaches a steady state, and solitons are periodically generated at the site of the bump and radiated upstream. However, it has not been known whether the phenomenon occurs for every $F$ in $\left(F_{L}, F_{C}\right)$. Our preliminary numerical results suggest that the periodic soliton generation occurs for every $F$ in a sub-interval of $\left(F_{L}, F_{C}\right)$. But, this sub-interval has not yet been determined.

Using $F_{C}$ is called the supercritical turning point since the upper branch and the lower branch of solitary waves merge into one at this point. $F_{L}$ is referred to as the subcritical cut-off point since the unique subcritical cnoidal waves change to a hydraulic fall at this point and the steady-state subcritical solution ceases to exist beyond this point.

If the formal asymptotic approach is valid, then the error of using the fKdV to describe the waves is of order $\varepsilon^{2}$. However, the validity of the formal $\mathrm{fKdV}$ asymptotics for this problem has not been proved even for the simplest possible situation: steady-state flow in a two-dimensional channel.

In the last ten years or so, it has been a noteworthy question whether the fKdV is 
an accurate mathematical model equation for the forced flow described above. If it is an accurate model for small $\varepsilon$, how big can the bump height, which is of order $\varepsilon^{2}$, be? It is well known that the $\mathrm{fKdV}$ is the result of the first-order approximation to the free-surface profile. Hence, the error is of order $O\left(\varepsilon^{2}\right)$. When $\varepsilon$ is very small, for instance, if $\varepsilon=0.3$, the error is of order $\varepsilon^{2}=0.09=9 \%$. The exact error depends on the coefficient of $\varepsilon^{2}$ in the error expression. If the coefficient is 5 , then the error for $\varepsilon=0.3$ is $45 \%$, which is in general too big to be accepted for laboratory experimental studies. For the same coefficient 5 , the error is $5 \%$ when $\varepsilon=0.1$. In this case the height of the bump is very small (of order $\varepsilon^{2}=1 \%$ of the depth) and the error is also small $(5 \%)$. Hence one may infer that the $\mathrm{fKdV}$ is a valid model for very small $\varepsilon$, i.e., for a very small bump. However, if the bump height is restricted to the order of $1 \%$ of the depth, there is little significance for the fKdV modeling, for the free surface would not feel the disturbance caused by such a small bump on the bottom. The experiments were all conducted for a bump height not less than one tenth of the depth. Otherwise, the unusual phenomena associated solely with the forced system cannot be clearly observed. Does this tell us that the fKdV theory is a dead end for the mathematical modeling of our forced-surface waves?

Surprisingly enough, the answer is NO in spite of the facts listed in the above paragraph. The validity range of the $\mathrm{fKdV}$ is much wider. The error in the $\mathrm{fKdV}$ asymptotic result is less than $10 \%$ when $\varepsilon$ is less than 0.7 [6]. However, when $\varepsilon>0.7$, the error increases so dramatically that the $\mathrm{fKdV}$ becomes invalid.

In this paper an asymptotically reduced model is defined to be good if the difference (i.e., error) between results of the model and the experiment is less than $10 \%$. By comparing the result from the fKdV theory and those from the direct numerical integration of the Laplace equation (called the computational method) and experiments, we will demonstrate that the stationary fKdV is still a good model up to $\varepsilon=0.7$ for semicircular bumps. The bump height now is about $50 \%$ of the upstream uniform depth. Examining the reports from experiments and the compurational approach, the fKdV is a good model when the bases of the bumps are short, when the experiments can be conducted and when the computational method is convergent.

In 1989, Lee et al. made an extensive quantitative comparison between the fKdV theory and the experiments for transcritical upstream soliton radiations [7]. The flow under their consideration is intrinsically unsteady and the amplitude of the forcing is less than $20 \%$ of the depth. Their comparison implied that the agreement between the fKdV theory and the experiments is generally very good, in terms of both the amplitude and the period of the soliton generation, especially at the critical and nearcritical speeds. As the Froude number departs from 1, the fKdV theory increasingly overestimates but only slightly the experiments. This was the first time people showed the validity of the fKdV. Since there have not yet been results available from direct numerical integration of the Laplace equation for time-dependent flows, it is still impossible to compare the $\mathrm{fKdV}$ model at the transcritical range with computational results from the direct numerical integration of the Laplace equation.

Comparisons were recently made by Shen [6] of stationary supercritical multiple solitary waves, stationary subcritical cnoidal waves, and hydraulic falls in the case 
of short bumps (he called it local forcing). In the present paper, we provide more comparisons between the $\mathrm{fKdV}$ model and computational and experimental results of stationary flows. Most of the comparisons here are made for long bumps (Shen called it non-local forcing [6]). Our results again imply that the fKdV model is good only when the bump height is less than one half of the upstream depth. When the bump height is larger than one half of the depth and the base of the bump is very long, the fKdV is not a valid model and the Green-Naghdi model may be applicable.

The major novel points of the present paper consist of: (i) a new and simple derivation of the stationary $\mathrm{fKdV}(\mathrm{sfKdV})$; (ii) a proof of the existence theorem for the solitary wave solutions of the sfKdV; (iii) a systematic study of the accuracy of the sfKdV as a model equation; (iv) the analysis on the error due to the local forcing assumption and the elucidation on the different nature of the $\mathrm{fKdV}$ modeling from the $\mathrm{KdV}$ modeling. Although part of the materials in [6] delineate the error problem for the fKdV modeling, most of the above four points are not covered there.

The content of this paper is arranged as follows. In Sec. 2, we give a new and more rigorous derivation of the stationary fKdV. The existence and properties of the solutions to this stationary fKdV are discussed in Sec. 3. The extensive comparison of results from sfKdV, computation and experiment is described in Secs. 4 and 5. In Sec. 6, we draw our conclusions and present some discussions on when to use the fKdV.

2. Derivation of the stationary forced Korteweg-de Vries equation. The fluid under consideration is inviscid and incompressible. The fluid flow configuration is shown in Fig. 1(a). Let the $x^{*}$-axis be aligned along the longitudinal direction and on the bottom of the channel, and the $y^{*}$-axis be vertically opposite to the gravitational direction. The quantity $H$ is the upstream depth of the fluid, $\rho$ the density, $p^{*}$ the pressure field, $\left(u^{*}, v^{*}\right)$ the velocity field, $\eta^{*}$ the free-surface elevation, and $c^{*}$ the upstream uniform velocity. The function $y^{*}=h^{*}\left(x^{*}\right)$ represents the bottom topography and $g$ is the gravitational acceleration.

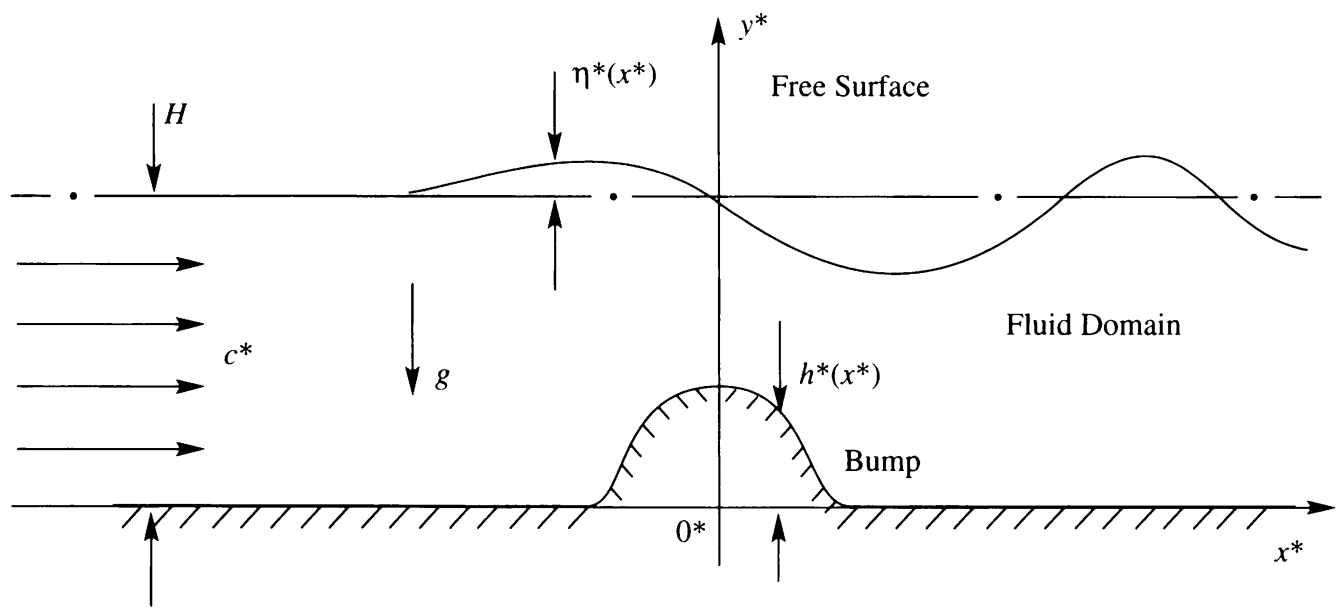

FIG. 1 (a) Configuration of a steady-state fluid flow with free surface over a bump in the laboratory coordinate system 
Let $L$ be the typical wave length, which is yet to be determined. Meantime, $L$ is assumed to be much larger than $H$ in the long-wave assumption. We use $L$ and $H$ as the horizontal and vertical scales, respectively. We use $\rho$ as the density scale. The following dimensionless variables were introduced:

$$
\begin{aligned}
& \varepsilon=(H / L)^{2} \ll 1 \text { (a small parameter in the } \mathrm{fKdV} \text { asymptotic analysis), } \\
& (x, y)=\left(\varepsilon^{1 / 2} x^{*}, y^{*}\right) / H, \\
& p=p^{*} /(\rho g H), \quad(u, v)=\left(u^{*}, \varepsilon^{-1 / 2} v^{*}\right) / \sqrt{g H} \\
& F=c^{*} / \sqrt{g H} \text { (Froude number), } \quad \eta=\eta^{*} / H \\
& h(x)=\varepsilon^{-2} h^{*}\left(x^{*}\right) / H \text { (small bump assumption). }
\end{aligned}
$$

Since the flow is laminar, both the free surface and the bottom of the channel are stream lines. Let $\psi$ be the dimensionless stream function such that $\psi=1$ on the free surface and $\psi=0$ on the bottom. Then

$$
u=F \frac{\partial \psi}{\partial y}, \quad v=-F \frac{\partial \psi}{\partial x} .
$$

We make the following coordinate transformation: $(x, y) \rightarrow(\zeta, \psi)$ such that $\zeta=x, \psi=\psi(x, y)$. Hence $(\zeta=x, \psi=\psi(x, y))$ maps the fluid domain in the $(x, y)$-plane onto a strip $\Omega=\mathfrak{R} \times(0,1)$ in the $(\zeta, \psi)$-plane (see Fig. $1(\mathrm{~b}))$. Here $(\zeta, \psi)$ is called the streamline coordinate system. Let $(x-\zeta, y=f(\zeta, \psi))$ be the inverse transformation of $(x, y) \rightarrow(\zeta, \psi)$. In terms of the streamline coordinates $(\zeta, \psi)$, we have

$$
\begin{gathered}
u=\frac{F}{f_{\psi}}, \quad v=F \frac{f_{\zeta}}{f_{\psi}}, \\
\frac{\partial}{\partial x}=\frac{\partial}{\partial \zeta}-\frac{v}{F} \frac{\partial}{\partial \psi}, \quad \frac{\partial}{\partial y}=\frac{u}{F} \frac{\partial}{\partial \psi} .
\end{gathered}
$$

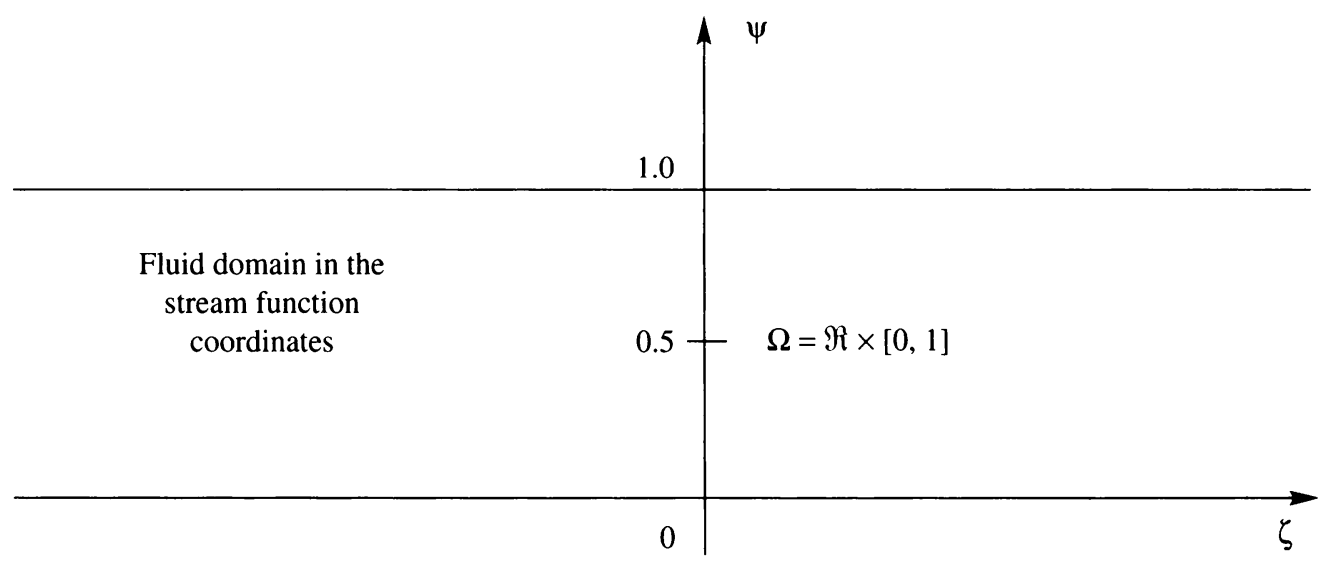

FIG. 1 (b) The fluid domain in the stream function coordinate system 
From the irrotationality condition $u_{y}-\varepsilon v_{x}=0$, one can derive a second-order partial differential equation for $f$ with independent variables $\zeta$ and $\psi$ in $\Omega$. Since the stream function is used, the kinematic boundary conditions on both the free surface and the bottom are satisfied automatically. Hence the Bernoulli equation shall serve as the boundary condition on $\psi=1$ and the bottom topography shall serve as the boundary condition on $\psi=0$. The resulting boundary-value problem is posed by

$$
\begin{aligned}
\varepsilon f_{\psi}^{2} f_{\zeta \zeta}-2 \varepsilon f_{\zeta} f_{\psi} f_{\zeta \psi}+\left(1+\varepsilon f_{\zeta}^{2}\right) f_{\psi \psi} & =0 & & \text { in } \Omega, \\
F^{2}\left(1+\varepsilon f_{\zeta}^{2}\right)+\left[2(f-1)-F^{2}\right] f_{\psi}^{2} & =0 & & \text { on } \psi=1, \\
f & =\varepsilon^{2} h(\zeta) & & \text { on } \psi=0 .
\end{aligned}
$$

This procedure was used in 1960 by Peters and Stoker [8]. Nevertheless, this method appears to be applied to forced water wave motion in the context of the fKdV for the first time. Let $F_{0}$ be the critical Froude number. It is assumed that the upstream velocity is near the critical speed and the response of the free-surface elevation to the bump is of $O(\varepsilon)$. Hence we have the following asymptotic expansion:

$$
\begin{aligned}
& f=\psi+\varepsilon f_{1}(\zeta, \psi)+\varepsilon^{2} f_{2}(\zeta, \psi)+O\left(\varepsilon^{3}\right), \\
& F=F_{0}+\varepsilon \lambda+O\left(\varepsilon^{2}\right) .
\end{aligned}
$$

Substituting Eqs. (7) and (8) into Eqs. (4)-(6), one can obtain a sequence of equations of successive orders. We need to use only those equations of the first two orders.

The problem of $O(\varepsilon)$ order:

$$
\begin{aligned}
f_{1 \psi \psi}=0 & \text { in } \Omega, \\
f_{1}-F_{0}^{2} f_{1 \psi}=0 & \text { on } \psi=1, \\
f_{1}=0 & \text { on } \psi=0 .
\end{aligned}
$$

The problem of $O\left(\varepsilon^{2}\right)$ order:

$$
\begin{aligned}
f_{2 \psi \psi} & =-f_{1 \zeta \zeta} & & \text { in } \Omega, \\
f_{2}-F_{0}^{2} f_{2 \psi} & =2 F_{0} \lambda f_{1 \psi}-2 f_{1} f_{1 \psi}+\left(F_{0}^{2} / 2\right) f_{1 \psi}^{2} & & \text { on } \psi=1, \\
f_{2} & =h(\zeta) & & \text { on } \psi=0 .
\end{aligned}
$$

Equations (9)-(11) imply that

$$
f_{1}=\psi A(\zeta), \quad F_{0}^{2}=1 \quad \text { (the critical speed of the shallow water waves) }
$$

where $A$ is an arbitrary function of $\zeta$ to be determined by the solvability condition of the second-order problem (12)-(14). Since $f_{1}(\zeta, \psi=1)=A(\zeta=x)$, thus $A(x)$ is the first-order elevation of the free surface.

Multiplying (12) by $f_{1}$ and integrating the resulting equation with respect to $\psi$ from $\psi=0$ to 1 , one can obtain that

$$
A^{\prime \prime}-6 \lambda A=-\frac{9}{2} A^{2}-3 h
$$


This is the first integral of the well-known stationary fKdV

$$
\lambda A_{x}-\frac{3}{2} A A_{x}-\frac{1}{6} A_{x x x}=\frac{1}{2} h_{x}
$$

under the condition that the far upstream flow is uniform (i.e., $A(-\infty)=A^{\prime}(\infty)=$ $A^{\prime \prime}(-\infty)=0$ and $c^{*}=$ constant $)$.

3. Existence of positive solitary wave solutions. In this section, we prove the existence of solutions to the following sfKdV boundary-value problem:

$$
\begin{gathered}
\lambda v(x)+\alpha v^{2}(x)+\beta v^{\prime \prime}(x)=f(x), \\
v( \pm \infty)=v^{\prime}( \pm \infty)=0,
\end{gathered}
$$

where $\alpha<0, \beta<0, \lambda>0$, and the forcing $f(x)$ is nonnegative with $\operatorname{supp}(f)=$ $\left[x_{-}, x_{+}\right]$.

The proof here is based on the use of the monotone iteration scheme and is different from that given in [5] or [6]; the latter uses the contraction mapping theorem and the analytic extension of bifurcation branches.

We first convert the BVP (17)-(18) into an (integral) operator equation $v=T(v)$. Then, we find an upper solution $u^{0}$ and a lower solution $u_{0}$. Through the standard monotone iteration procedure [9], the existence of a maximal solution $\bar{u}$ and a minimal solution $\underline{u}$ in $\left[u_{0}, u^{0}\right]$ is established.

The operator equation obtained from the BVP (17)-(18) is

$$
v(x)=\frac{1}{\beta} \int_{-\infty}^{\infty} K(x, \xi)\left(\alpha v^{2}-f\right)(\xi) d \xi \equiv T(v)(x)
$$

where

$$
K(x, \xi)=\frac{1}{2 \nu} \exp (-\nu|\xi-x|)
$$

with $\nu=\sqrt{-\lambda / \beta}$.

In the following, we say that $u \geq v$ for $u \in L^{2}(-\infty, \infty)$ and $v \in L^{2}(-\infty, \infty)$ if $u(x) \geq v(x)$, a.e. This ordering is denoted by $u \geq v$. To use the monotone iteration scheme, we need to find an upper solution and a lower solution. By definition, $u_{0}$ is a lower solution of (19) if $u_{0} \leq T\left(u_{0}\right)$ and $u_{0}$ is an upper solution of (19) if $u^{0} \geq T\left(u^{0}\right)$. It is clear that $u_{0}=0$ is a lower solution of (19) since

$$
T\left(u_{0}=0\right)=-\frac{1}{\beta} \int_{-\infty}^{\infty} K(x, \xi) f(\xi) d \xi>0 \equiv u_{0} .
$$

Here the nonnegativity of $f(x)$ is used.

For an upper solution, we have the following lemma.

LEMMA. When

$$
\left(x_{+}-x_{-}\right)^{4}\|f\|_{\infty}<-57 \beta^{2} / \alpha,
$$

there exists an interval $\left(\lambda_{1}, \lambda_{2}\right) \subset(0, \infty)$ such that

$$
u^{0}(x)=\frac{1}{2}\left[-\frac{3 \lambda}{2 \alpha} \operatorname{sech}^{2}\left(\sqrt{\frac{-\lambda}{4 \beta} x}\right)\right]
$$


is an upper solution of (19) when $\lambda \in\left(\lambda_{1}, \lambda_{2}\right)$.

Proof of the lemma. The function

$$
s(x)=-\frac{3 \lambda}{2 \alpha} \operatorname{sech}^{2}\left(\sqrt{\frac{-\lambda}{4 \beta}} x\right)
$$

is a solution of the BVP (17)-(18) when $f(x) \equiv 0$ (unforced). Hence, we have

$$
\frac{\alpha}{\beta} \int_{-\infty}^{\infty} K(x, \xi) s^{2}(\xi) d \xi=s(x) .
$$

Now, let us compute $T\left(u^{0}\right)$ :

$$
\begin{aligned}
T\left(u^{0}\right)(x) & =\frac{1}{\beta} \int_{-\infty}^{\infty} K(x, \xi)\left(\alpha s^{2} / 4-f\right)(\xi) d \xi \\
& =\frac{\alpha}{4 \beta} \int_{-\infty}^{\infty} K(x, \xi) s^{2}(\xi) d \xi-\frac{1}{\beta} \int_{-\infty}^{\infty} K(x, \xi) f(\xi) d \xi \\
& =\frac{s(x)}{4}-\frac{1}{\beta} \int_{-\infty}^{\infty} K(x, \xi) f(\xi) d \xi \\
& =\frac{1}{2} u^{0}(x)-\frac{1}{\beta} \int_{-\infty}^{\infty} K(x, \xi) f(\xi) d \xi
\end{aligned}
$$

To make $u^{0}=s / 2$ an upper solution, we need

$$
u^{0} \geq T\left(u^{0}\right)
$$

This is equivalent to

$$
\frac{1}{4}\left(-\frac{3 \lambda}{2 \alpha}\right) \operatorname{sech}^{2}\left(\frac{\nu}{2} x\right) \geq-\frac{1}{2 \nu \beta} \int_{x_{-}}^{x_{+}} \exp (-\nu|\xi-x|) f(\xi) d \xi .
$$

This inequality holds when the conditions of the lemma are satisfied. Thus $u^{0}=$ $s(x) / 2$ is an upper solution of (19) when $\lambda \in\left(\lambda_{1}, \lambda_{2}\right)$. This finishes the proof of the lemma.

Theorem 1. The problem (17)-(18) has at least one positive solution when the conditions of the lemma are satisfied.

Proof. We prove this theorem by the monotone iteration procedure. First of all, we show that $T$ is a strictly increasing operator in $L_{N}^{2}(-\infty, \infty)=\{u \mid u \in$ $L^{2}(-\infty, \infty), 0 \leq u(x) \leq N$ a.e. for a given positive number $\left.N\right\}$. Let $u, v \in$ $L_{N}^{2}(-\infty, \infty)$ and $u>v$. The following expression,

$$
T(u)(x)-T(v)(x)=\frac{\alpha}{\beta} \int_{-\infty}^{\infty} \frac{1}{2 \nu} \exp (-\nu|\xi-x|)(u+v)(u-v)(\xi) d \xi>0,
$$

implies that $T(u)>T(v)$. Hence, $T$ is strictly increasing.

Secondly, we show that $T$ is continuous in $L_{N}^{2}(-\infty, \infty)$. Let $\left\{w_{n}\right\}_{n=1}^{\infty}$ be a sequence in $L_{N}^{2}(-\infty, \infty)$, and $\lim _{n \rightarrow \infty} w_{n}=w \in L_{N}^{2}(-\infty, \infty)$. By the Lebesgue 
dominant convergence theorem, we have

$$
\begin{aligned}
\lim _{n \rightarrow \infty} T\left(w_{n}\right)(x) & =\lim _{n \rightarrow \infty} \frac{1}{\beta} \int_{-\infty}^{\infty} \frac{1}{2 \nu} \exp (-\nu|\xi-x|)\left(\alpha w_{n}^{2}-f\right)(\xi) d \xi \\
& =\frac{1}{\beta} \int_{-\infty}^{\infty} \frac{1}{2 \nu} \exp (-\nu|\xi-x|)\left(\alpha \lim _{n \rightarrow \infty} w_{n}^{2}(\xi)-f(\xi)\right) d \xi \\
& =T(w) .
\end{aligned}
$$

Hence, $T$ is continuous.

Thirdly, we construct iteration sequences. Let

$$
u_{n}=T\left(u_{n-1}\right), \quad n=1,2,3, \ldots
$$

Since $T$ is increasing, applying $T$ to the following inequality,

$$
u_{1}=T\left(u_{0}\right) \geq u_{0}=0 \text {, }
$$

we have

$$
u_{2}=T\left(u_{1}\right) \geq T\left(u_{0}\right)=u_{1} .
$$

Repeating the same procedure, one obtains

$$
u_{0} \leq u_{1} \leq u_{2} \leq u_{3} \leq \cdots \text {. }
$$

Similarly, we define

$$
u^{n}=T\left(u^{n-1}\right), \quad n=1,2,3, \ldots
$$

and obtain

$$
u^{0} \geq u^{1} \geq u^{2} \geq u^{3} \geq \cdots .
$$

To find the bounds of the sequences (29) and (31), we apply $T$ to the inequality $u_{0}<u^{0} \quad n$ times and obtain $T^{n}\left(u_{0}\right)<T^{n}\left(u^{0}\right), n=1,2,3, \ldots$ This implies that

$$
u_{0} \leq u_{1} \leq u_{2} \leq \cdots \leq u_{n}<\cdots<u^{n} \leq \cdots \leq u^{2} \leq u^{1} \leq u^{0} .
$$

Since $u_{n}(x)$ is an increasing sequence of differentiable functions bounded from above, its pointwise convergence immediately follows. This implies the existence of the maximal solution $\bar{u}(x)=\lim _{n \rightarrow \infty} u^{n}(x)$. The argument for the existence of the minimal solution is similar. The proof of the theorem is complete.

It is worth making the following remarks about the theorem to avoid possible confusion with the multiplicity of the solutions of the $\mathrm{sfKdV}$. It is this multiplicity that makes the research on the sfKdV interesting. The conditions of the theorem are only sufficient for the existence of solitary wave solutions of the sfKdV. They are by no means the necessary conditions. Both numerical simulations for non-local forcings and analytic solutions for local forcings show that solutions always exist for large values of $\lambda[5,6]$. Hence, solutions can still exist when $\lambda$ is not necessarily in $\left(\lambda_{1}, \lambda_{2}\right)$. In addition, the existence of the maximal and minimal solutions does not imply that there are only two solutions. The maximal and minimal solutions may coincide with each other in one case but there may exist solutions that are not ordered relative to the maximal and minimal solutions in other cases. 
4. Choice of $\varepsilon$ and $\mathbf{s f K d V}$ in laboratory coordinates. It appears that no analytic approach is possible to check the accuracy of the sfKdV derived in Sec. 2 as a mathematical model equation. One thus needs to take the approach that compares the results obtained from the sfKdV model with those obtained from experiment and computational method. The comparison must be made in the same coordinate system. For the sake of convenience in handling the experimental data, we choose the laboratory coordinates as our working coordinate system in this paper. Therefore, we will calibrate all the data according to the laboratory scale. For the sfKdV asymptotic model, the basic concern is the shrinking of the horizontal length scale because of the long wave assumption for the sfKdV. The vertical scale is taken to be the same as the horizontal scale in the computational approach in all the published literature.

The inverse operation of the nondimensionalization procedure at the beginning of Sec. 2 enables us to bring the sfKdV (16) back to the sfKdV in laboratory scales:

$$
H^{3} A_{x^{*} x^{*}}^{*}-6 \sqrt{\frac{H}{g}} u_{\lambda}^{*} A^{*}=-\frac{9}{2}\left(A^{*}\right)^{2}-3 H h^{*}\left(x^{*}\right) .
$$

Here $u_{\lambda}^{*}=\varepsilon \lambda \sqrt{g H}=c^{*}-\sqrt{g H}$ is the perturbation of the upstream velocity from its critical value and $A^{*}\left(x^{*}\right)=\varepsilon H A\left(x^{*} / L\right)$ describes the free-surface profile in the laboratory frame.

For a given upstream Froude number $F$, the perturbed upstream velocity is expressed by $u_{\lambda}^{*}=(F-1) \sqrt{g H}$. Hence Eq. (33) is independent of the small number $\varepsilon$. To find a profile of the free surface, one can solve the following IVP of an ODE:

$$
\begin{gathered}
H^{3} A_{x^{*} x^{*}}^{*}-6 H(F-1) A^{*}=-\frac{9}{2}\left(A^{*}\right)^{2}-3 H h^{*}\left(x^{*}\right), \\
A^{*}(-\infty)=A_{x^{*}}^{*}(-\infty)=0 .
\end{gathered}
$$

Analytic solutions of the above IVP for a general $h^{*}\left(x^{*}\right)$ have been found for certain special types of forcing functions [2,7], but not for a general forcing function $h^{*}\left(x^{*}\right)$. The interval in which we have difficulty finding analytic solutions of the $\operatorname{sfKdV}$ is $\operatorname{supp}(h)$. Therefore a numerical method has to be applied. Nonetheless, if the interval length of $\operatorname{supp}(h)$ is very short and approximately regarded as zero, then analytic solutions from two sides of $\operatorname{supp}(h)$ can be matched on $\operatorname{supp}(h)$, which is now regarded as a single point. This is the case when $\varepsilon$ is very small (hence the bump height is very small) and the base of the bump is not larger than twice the bump height, and the forcing term $h(x)$ in the dimensionless $\mathrm{sfKdV}(16)$ has the properties that (i) $\max h(x)=1$ if $\varepsilon^{2}=\max h^{*}\left(x^{*}\right) / H$, and (ii) the graph of $h(x)$ is very slim (see Fig. 2 (b)). This slim graph can be further approximated by $Q \delta(x)$ where $\delta(x)$ is the Dirac delta function under the condition that $Q$ is equal to the area under the slim graph. This procedure is a pure mathematical approximation and has no physical meaning. Solutions of the dimensionless $\mathrm{fKdV}$ (16) can now be found analytically and hence the results become more transparent $[6,10]$. The price paid for this approximation is that the mathematical solution of the $\mathrm{fKdV}$ is no longer smooth at $x=0$.

The first step in the $\delta$-function approximation is to choose a value for $\varepsilon$, which should be determined by the nature of the physical assumptions. For the problem 


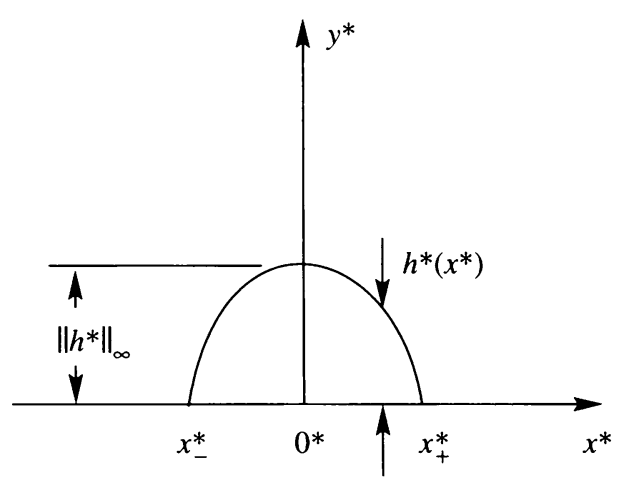

(a)

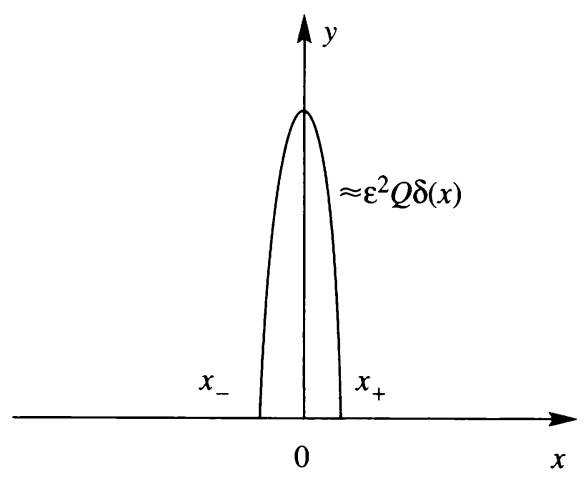

(b)

FIG. 2 (a) A bump with a short base before stretching in the laboratory coordinates; (b) the same bump after stretching in dimensionless sfKdV coordinates. Here $\varepsilon=\left\|h^{*}\right\|_{\infty} / H, x_{ \pm}=\varepsilon^{1 / 2} x_{ \pm}^{*} / H, S=$ $\int_{x_{-}^{*}}^{x_{+}^{*}} h^{*}\left(x^{*}\right) d x^{*}$, and $Q=\varepsilon^{-3 / 2} S / H^{2}$.

under investigation, the nature of the physical assumptions in setting up the experimental apparatus is reflected in the assumption that the bump height is of order $\varepsilon^{2}$ relative to the upstream depth $H$.

The function $y^{*}=h^{*}\left(x^{*}\right)$ describes the shape of the bump (see Fig. 2(a)). In terms of the dimensionless $\mathrm{fKdV}$ asymptotic variables, the bump is $y=\varepsilon^{2} h(x)$ as shown in Fig. 2(b). As an example, let us look at the semicircle bump: $y^{*}=$ $\sqrt{R^{2}-x^{* 2}}$. In dimensionless $\mathrm{fKdV}$ asymptotic scales, $x=\varepsilon^{1 / 2} x^{*} / H, y=y^{*} / H$, and the semicircular bump becomes $y=(1 / \sqrt{\varepsilon}) \sqrt{\varepsilon \alpha^{2}-x^{2}}$, where $\alpha=R / H$. The support of the bump is now $[-\sqrt{\varepsilon} \alpha, \sqrt{\varepsilon} \alpha]$, which is $\sqrt{\varepsilon}$ times smaller than the dimensionless height $\alpha$. If $\varepsilon$ is very small, the support of $h(x)$ is very small. Hence $h(x)$ can be approximated by $Q \delta(x)$. This height $\alpha$ can be regarded as the amplitude of the forcing. Hence $\varepsilon^{2}=\alpha$, i.e.,

$$
\varepsilon=\sqrt{\alpha} .
$$

In the $(x, y)$ dimensionless coordinate system, the bump becomes a slim ellipse $y=\varepsilon^{2} \sqrt{1-(x / \sqrt{\varepsilon} \alpha))^{2}}$. Hence, $h(x)=\sqrt{1-(x /(\sqrt{\varepsilon} \alpha))^{2}}$. The area under the delta function should be the same as that under $h(x)$. The area inside the semi-ellipse whose two semiaxes are 1 and $\sqrt{\varepsilon} \alpha$ is $\pi \cdot 1 \cdot \sqrt{\varepsilon} \alpha / 2=\pi \alpha^{5 / 4} / 2$. Therefore,

$$
Q=(\pi / 2) \alpha^{5 / 4} \text {. }
$$

As a matter of fact, there are infinitely many ways to choose $\varepsilon$ and $Q$. Yet, according to [6] the physical quantities derived from the asymptotic analysis are the same in spite of different values of $\varepsilon$ and $Q$. For example, the important quantities $F_{C}$ and $F_{L}$ mentioned in Sec. 1 are always given by

$$
\begin{aligned}
& F_{C}=1+\frac{1}{2}\left(9 S /\left(4 H^{2}\right)\right)^{2 / 3}, \\
& F_{L}=1-\frac{1}{2}\left(9 S /\left(2 H^{2}\right)\right)^{2 / 3}
\end{aligned}
$$

where $S=\int_{-\infty}^{\infty} h^{*}\left(x^{*}\right) d x^{*}$ is the area of the bump. 
5. Comparison of the results from the sfKdV, experiments and computations. We are now ready to compare the results obtained from the $\mathrm{fKdV}$ model and those from experiments and direct numerical integration of the Laplace equation (called the computational method). The experimental data used here is from Sivakumaran et al. ([11] or [12]). The computational data is from Dias and Vanden-Broeck [13].

5.1. The differences between the $K d V$ model and the fKdV model. Upon discovering the soliton solutions of the Korteweg-de Vries equation (KdV) in 1965, people were concerned with the accuracy of the KdV model when it is used as a model equation to describe certain physical phenomena, such as the long surface waves in a channel. Hammack and Segur carried out extensive experimental studies to justify the validity of the $\mathrm{KdV}$ model and concluded that [14]

the Korteweg-de Vries equation appears to provide an accurate model for determining the evolution from various sets of initial data of gravity waves of moderate amplitude propagating in one direction in a non-dissipative or slightly dissipative fluid of uniform depth.

We intend to point out some substantial differences between the KdV modeling and the fKdV modeling in terms of the setup of experiments and the determination of the values of the small parameter $\varepsilon$.

The most notable type of solution of the $\mathrm{KdV}$ initial value problem is the $\mathrm{KdV}$ soliton. The KdV solitons are a train of solitons with unequal amplitudes. The total number of solitons generated depends on both the size and the shape of the initial pile of mass. This pile of mass can be accumulated in different ways. Hammack and Segur's way is to push a pile of water mass up from the bottom at the left end of the water tank by a piston. Let $N$ be the total number of solitons generated by this pile of water. The volume of the water mass of the $k$ th soliton is $4 k H L B(k=$ $1,2, \ldots, N)$, where $H$ is the depth of the water at rest, $L$ is the horizontal length scale, and $B$ is the width of the water tank. The volume of this pile of water must be greater than or equal to $N(N+1) H L B=\sum_{k=1}^{N} 4 k H L B$. Hence, it is appropriate to determine the value of $\varepsilon$ by the initial condition. In [14], the authors chose $\varepsilon=\left\|\eta_{0}^{*}\right\|_{\infty} / H$, where $\eta_{0}^{*}(x)$ is the initial profile of the free surface (i.e., $\left.\eta_{0}^{*}(x)=\eta^{*}(x, t=0)\right)$.

In the asymptotic expansion, $\varepsilon$ is a constant throughout the whole process. Because of the inevitable presence of small viscosity in the water tank experiments such as those conducted by Hammack and Segur, the memory of the physical system of the initial condition gradually fades out and consequently diminishes the value of $\varepsilon$. Thus, $\varepsilon$ equal to 0.1 at time equal to $5.0 \sqrt{\mathrm{H} / \mathrm{g}}$ implies a larger value of $\varepsilon$ at time $0.0 \sqrt{H / g}$. This can be one of many factors that may imply the consequence of error growing with time. Hence, the $\mathrm{KdV}$ modeling approach has been suspect due to viscosity effects when its results are compared with experiment. To make a fair comparison between the $\mathrm{KdV}$ asymptotic results and the experimental results, one must make some adjustment to the asymptotic results because of the viscosity. Hammack and Segur [14] adapted the adjustment scheme due to Keulgan (1948). With this adjustment, they showed that the error in the KdV model is within $20 \%$ when $\varepsilon<0.44$. 
The setup of experiments for the validation of the $\mathrm{fKdV}$ model is quite different from that for the KdV model described above. For the $\mathrm{fKdV}$, either there is a uniform upstream current coming in from one end and going out at the other end of the tank, or the bump (the forcing) on the bottom moves at a constant speed in one direction. As shown in the last section, the small number $\varepsilon$ is now determined by the size of the bump: $\varepsilon=(R / H)^{1 / 2}$ for a semicircular bump and $\varepsilon=\left(S / H^{2}\right)^{1 / 4}$ in general. Since the size of a bump is a boundary condition that does not change with respect to time, the small viscous dissipation does not lead to a decrease in the value of $\varepsilon$. Hence, no serious adjustment for viscosity is needed. Thus, one can expect better accuracy of the sfKdV model when considering surface waves. Much evidence supports this expectation. Therefore, regarding the accuracy of the asymptotics, to determine the value of $\varepsilon$ from the initial data for the $\mathrm{KdV}$ and to determine the value of $\varepsilon$ from the boundary data for the $\mathrm{fKdV}$ constitutes the major difference between the $\mathrm{KdV}$ modeling the the fKdV modeling. The above qualitative argument provides an explanation for the remarkable accuracy of the fKdV model.

The accuracy of the sfKdV as a model equation for the case of semicircular bump forcing was studied in [6]. The conclusion is that the error is with $10 \%$ when $\varepsilon<0.7$. In the following we examine the cases of triangular bumps and Gaussian bumps.

Let us investigate the case of triangular bumps first. Computational results used here are from [13]. The volume flux is denoted by $q$ and hence is a constant throughout the entire channel. The length scale used in [13] is, where the horizontal and vertical length scales are the same in their numerical computations, $\left(q^{2} / g\right)^{1 / 3}=\left[\left(F_{D} \sqrt{g H_{D}} H_{D}\right)^{2} / g\right]^{1 / 3}=F_{D}^{2 / 3} H_{D}$ and the velocity scale is $(q g)^{1 / 3}=$ $\left(F_{D} \sqrt{g H_{D}} H_{D} g\right)^{1 / 3}=F_{D}^{1 / 3} \sqrt{g H_{D}}$, where $H_{D}$ and $F_{D}$ are, respectively, the depth and the Froude number of the far downstream flow. The bumps considered in [13] are isosceles triangles with the top vertex a $90^{\circ}$ angle. The dimensionless bump height is $W$ and hence the laboratory height of the bump is $W F_{D}^{2 / 3} H_{D}$. The bump area is $S=\left(W F_{D}^{2 / 3} H_{D}\right)^{2}$. Figure 3 in [13] shows a relationship between $F_{D}$ and the size of the bump that is characterized by the bump height $W$. From (40), we have

$$
F_{L}=1-\frac{1}{2}\left(\frac{9}{2} W^{2} F_{D}^{4 / 3} H_{D}^{2} / H^{2}\right)^{2 / 3}
$$

The conservation of the mass flux implies that

$$
F_{L}=\left(H_{D} / H\right)^{3 / 2} F_{D}
$$

Using the Bernoulli equation, one can derive that

$$
\frac{H_{D}}{H}=\frac{\sqrt{8+F_{D}^{2}}-F_{D}}{2 F_{D}} .
$$


See Eq. (2.6) in [13]. From the above three expressions, one can obtain that

$$
W=\left(\frac{2^{9 / 8}}{3}\right) \frac{F_{D}^{1 / 3}\left[2^{3 / 2}-\left(\sqrt{8+F_{D}^{2}}-F_{D}\right)^{3 / 2} F_{D}^{-1 / 2}\right]^{3 / 4}}{\sqrt{8+F_{D}^{2}}-F_{D}}, \quad F_{D} \geq 1
$$

where $F_{D}$ is the downstream Froude number of the hydraulic fall. The comparison between the results of this formula derived from the sfKdV theory and those from numerical integration of the Laplace equation [13] is shown in Fig. 3 of this paper. Our result systematically underestimates $F_{L}$, and hence $F_{D}$. This is mainly due to the delta function approximation since this approximation does not distinguish the shape of the bump. Instead, it regards every bump as a very short obstruction of the given area. The shorter the bump, the better the approximation. Intuition suggests that the hydraulic fall over a shorter bump of the same area corresponds to a lower upstream velocity. This reason was pointed out recently in [6]. Despite this under-estimation of $F_{D}$, both the computational and the asymptotic approaches lead to a linear $W, F_{D}$ relationship when $F_{D}$ is larger than 1.5: Therefore, a linear correction term may be added to the asymptotic result. When $F_{D}>1.2$, we have the following correction:

$$
W_{D V} \approx W_{A}-\left(0.0467 F_{D}+0.0333\right)
$$

where $W_{D V}$ and $W_{A}$ are the values of $W$ obtained via a computational method (Dias and Vanden-Broeck [13]) and via an asymptotic method (Eq. (40) in this pa-

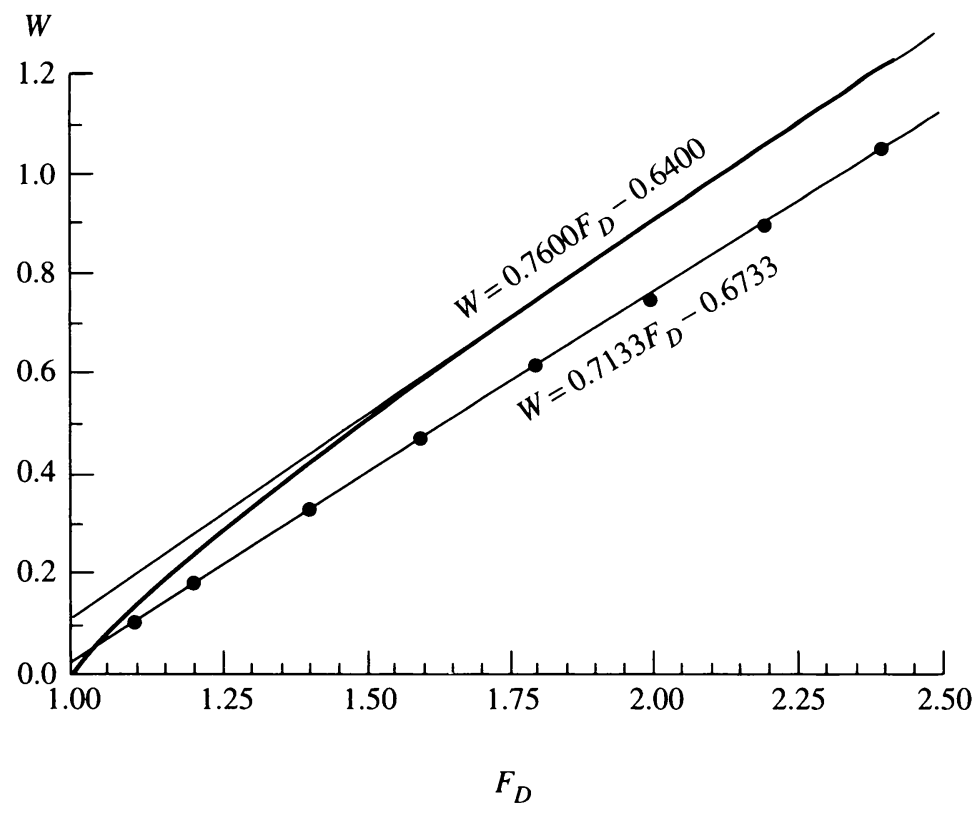

FIG. 3. The relationship between $W$ and $F_{D}$. The points represent the results from Dias and Vanden-Broeck [13] (see their Fig. 3). The thick curve represents the results from Eq. (41) of this paper. 
per), respectively. When $0 \leq F_{D} \leq 1.2$, the correction formula (43) fails to hold. After adjustment (43), the difference between the asymptotic results and the computational results is less than $10 \%$ except for a small neighborhood of $F_{D}=1.2$. Yet, in the laboratory experiment, hydraulic falls clearly show in the flow over a bump only when the downstream supercritical speed is reasonably large, e.g., $F_{D}$ is larger than 1.5. Next let us investigate the case of Gaussian bumps. Experimental results are taken from [11] (also see [12]). The bump considered in [11] is

$$
h^{*}\left(x^{*}\right)=20 \exp \left[-\frac{1}{2}\left(\frac{x^{*}}{24}\right)^{2}\right] \mathrm{cm} .
$$

The five hydraulic fall experiments reported by Sivakumaran et al in [11] are for upstream depth $H$ equal to $34.25,33.53,31.39,29.41,27.11 \mathrm{~cm}$, respectively. Hence the bump height is larger than one half of the upstream depth $H$. This implies that $\varepsilon$ is greater than 0.7. Consequently, as pointed out in [6] the sfKdV model loses its validity. Furthermore, the base of the bump is very long. So the delta function approximation also loses its validity.

Be that as it may, we still evaluate (40), which is based upon the sfKdV model and the delta function approximation. Surprisingly, both Sivakumaran et al's experimental results and our analytic result (40) yield a linear relationship between $F_{L}$ and $\alpha=20 / H$. As expected, our analytic result greatly underestimates $F_{L}$ (see Fig. 4 on p. 716). Let $F_{L}^{S T H}$ and $F_{L}^{S H N}$ denote the results from [11] and the present paper, respectively. Then there is the following correction relation:

$$
F_{L}^{S T H}=F_{L}^{S H N}-0.9931+2.671 \alpha .
$$

It is worth noticing that $F_{L}^{S H N}$ without correction does not have a physical meaning since $F_{L}^{S H N}$ is required to be positive.

For this large bump, the Green-Naghdi asymptotic theory [12] works much better than the sfKdV asymptotic theory. The Green-Naghdi theory yields the following governing equation for the free-surface profile $A^{*}$ :

$$
\begin{aligned}
A_{x^{*} x^{*}}^{*} & -\frac{1}{2} \frac{\left(A_{x^{*}}^{*}\right)^{2}}{H+A^{*}}+\frac{3}{2}\left(H+A^{*}\right)\left[\frac{1}{\left(H+A^{*}\right)^{2}}-\frac{1}{H^{2}}\right]+3 \frac{H+A^{*}}{F^{2} H^{3}} A^{*} \\
& =-3 \frac{H+A^{*}}{F^{2} H^{3}} h^{*}+\frac{3}{2}\left(h_{x^{*} x^{*}}^{*}+\frac{\left(h_{x^{*}}^{*}\right)^{2}}{H+A^{*}}\right) .
\end{aligned}
$$

Naghdi and Vongsarnpigoon showed that the results deduced from this theory agree with the experiments almost exactly (see their Fig. 2 in [12]). In the same figure, they showed that like the sfKdV theory, the hydrostatic theory also underestimates $F_{L}$. Although the sfKdV theory is not a hydrostatic theory, it is valid for only small or moderate free-surface elevation or depression. Actually, if we assume that $h^{*} / H=O\left(\left(A^{*} / H\right)^{2}\right), F=1+\left(A^{*} / H\right) \lambda$, and $(H / L)^{2}=O\left(\left(A^{*} / H\right)\right)$, then $\left(h_{x^{*}}^{*}\right)^{2}=$ $O\left(\left(A^{*} / H\right)^{3}\right), h_{x^{*} x^{*}}^{*} H=O\left(\left(A^{*} / H\right)^{3}\right)$, and $\left(A_{x^{*}}^{*}\right)^{2}=O\left(\left(A^{*} / H\right)^{3}\right)$. Consequently, the Green-Naghdi theory (44) yields

$$
H^{3} A_{x^{*} x^{*}}^{*}-6 H(F-1) A^{*}=-\frac{9}{2}\left(A^{*}\right)^{2}-3 H h^{*}\left(x^{*}\right)+O\left(\left(A^{*} / H\right)^{3}\right) .
$$




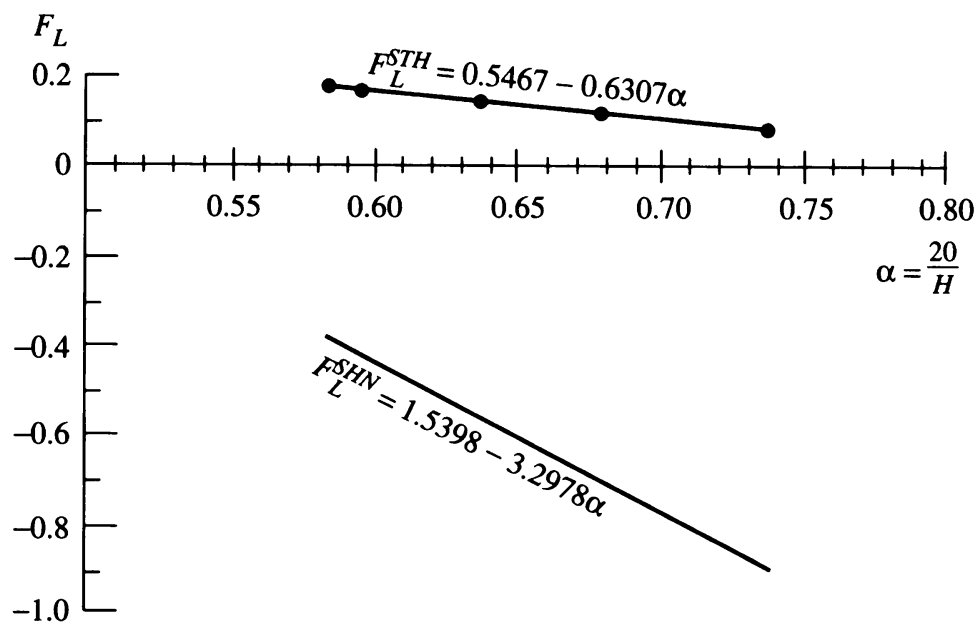

FIG. 4. The relationship between the dimensionless bump height $\alpha=20 / H$ and the cutoff Froude number $F_{L}$. The $F_{L}^{S T H}$ curve represents the results from Sivakumaran et al. [11] (see their Table 1). The $F_{L}^{S H N}$ curve represents the results from Eq. (40) of the present paper. The difference between the two straight lines is corrected by Eq. (44) of the present paper.

This is the $\mathrm{sfKdV}$ when $O\left(\left(A^{*} / H\right)^{3}\right)$ is ignored. Among the five experiments reported in [11], $\left\|A^{*}\right\|_{\infty} / H$ is as large as 0.84 for the first experiment. Such a large amplitude free-surface depression is beyond what the sfKdV can predict. Nevertheless, the $\mathrm{fKdV}$ theory can still qualitatively predict the solution behavior, in particular, the upstream Froude number $F_{L}$. In Table 1, we compare the experimental results reported in [11] and [12] (see Table 1 in [12]) with our results obtained by solving Eq. (46) numerically without the delta function approximation.

For a given Froude number and the bump defined by Eq. (43), to make the above comparison is to find the $H$ value. According to [12] (top three lines on p. 235), the physical meaning of $H$ is that $H$ is the minimum upstream depth that leads to a supercritical flow downstream. At this $H$ value, the upstream Froude number

TABLE 1. Comparison of the $H$ values at which the hydraulic falls occur. The experimental results are from Table 1 in [12] and the sfKdV results are obtained by solving (46) subject to boundary conditions: $A^{*}\left(x^{*}<0\right)=0$ and $A_{x}^{*}(\infty)=0$.

\begin{tabular}{|cccc|}
\hline$F^{2}$ & $H$ (experiments) & $H$ (sfKdV theory) & Relative error $(\%)$ \\
0.032 & 34.3 & 40.01 & 14 \\
0.028 & 33.6 & 39.25 & 14 \\
0.020 & 31.4 & 37.58 & 16 \\
0.013 & 29.4 & 35.89 & 18 \\
0.007 & 27.1 & 34.08 & 20 \\
\hline
\end{tabular}




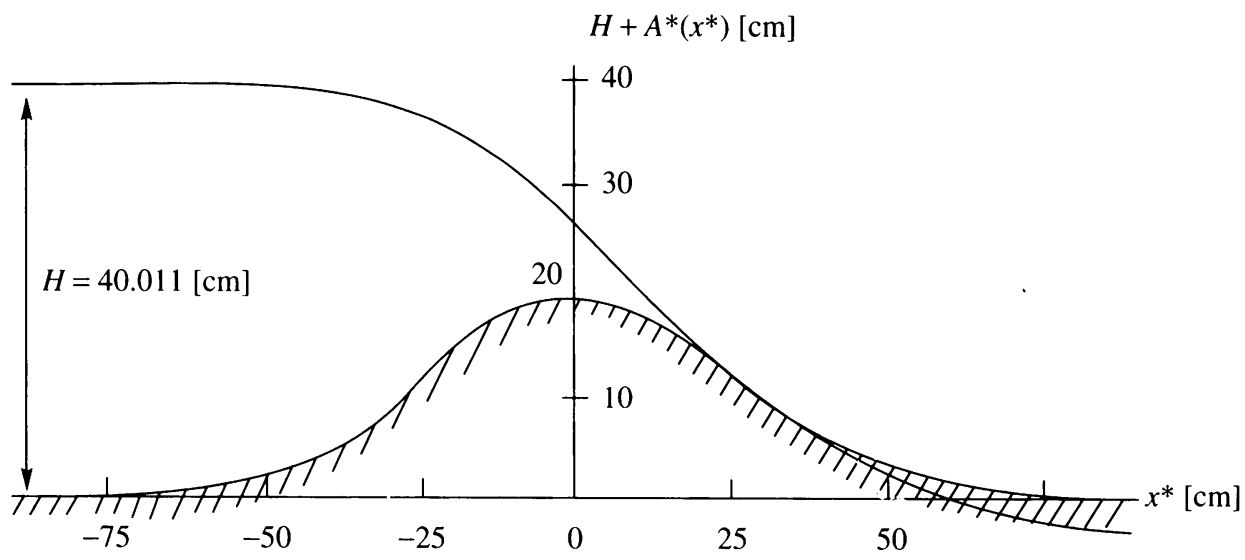

FIG. 5. The sfKdV fails to predict a correct free-surface profile of the hydraulic fall for a large long bump defined by Eq. (43). The upstream Froude number and depth are $F=\sqrt{0.032}$ and $H=40.011 \mathrm{~cm}$, respectively.

is $F_{L}$. When the upstream depth is greater than this value, the downstream flow is a cnoidal wave. When the upstream depth is smaller than this value and $F<F_{C}$, no steady-state flow is possible. As an example of showing flow profiles based upon the sfKdV theory, we plot the first case in Table $1: F^{2}=0.032$ and $H=40.011$ $\mathrm{cm}$ (see Fig. 5). Apparently, the flow profile downstream is unphysical since the free surface penetrates the rigid bottom! This example may suggest that although the $\mathrm{sfKdV}$ cannot predict the profile of the free surface for a very high and long bump, it still yields reasonable results on the upstream control parameter, the $H$ value or the $F_{L}$ value, when not using the delta function approximation.

Why did such a large amplitude free-surface depression as reported in [11] and [12] not break? This is because of the long base of the bump and upstream small Froude number. In contrast, for bumps with short bases, the failure of sfKdV yielding correct answers means that the bump is very high and the free surface will likely break because of a large velocity gradient in the flowing direction. The large velocity gradient leads to stronger nonlinearity. Eventually, the nonlinearity overwhelms the dispersion and free-surface waves break. The semicircular bump, for instance, belongs to this class of short base bumps, as pointed out in [6].

6. Conclusions. We have studied the errors due to the sfKdV asymptotic approximation. The sfKdV model, derived in the streamline coordinate system, is used to predict the subcritical cnoidal waves and hydraulic falls and supercritical solitary waves of order $O(\varepsilon)$ in steady-state flows. In the future, we intend to address the error size $O\left(\varepsilon^{2}\right)$ analytically from the formulation given in Sec. 2, i.e., to prove that $\left\|\eta^{*} / H-\varepsilon A\right\|=O\left(\varepsilon^{2}\right)$. Here the exact definition of the norm $\|\cdot\|$ is not yet known. When the forcing is nonnegative, sufficient conditions for the existence of the supercritical positive solitary wave solutions to the sfKdV are given in Sec. 3. They are not necessary conditions. Furthermore, numerical solutions for the sfKdV when the forcing is not nonnegative show that there exist several nonordered supercritical 
positive solitary wave solutions, yet a corresponding existence theorem has not been established.

The sf KdV model is simple. In particular, when the height of the bump is small and the base of the bump is short, the solutions can be found analytically. Consequently, the mechanism of the flow over a bump becomes transparent. From the derivation, one can see that the error in the $s f \mathrm{KdV}$ asymptotic approximation is of order $O\left(\left(\left\|\eta^{*}\right\|_{\infty} / H\right)^{2}\right)$. When the quantity $\left\|\eta^{*}\right\|_{\infty} / H$ is not small or moderate (when $\geq 0.7$ ), the sfKdV is most likely not a good model to be used in theoretical predictions. A higher order in the asymptotic approximation procedure should be included. One of the higher-order theories is the Green-Naghdi model where the error is of order $O\left(\left(\left\|\eta^{*}\right\|_{\infty} / H\right)^{3}\right)$. By comparing with experiment, it has been shown that the theory is still valid when the quantity $\left\|\eta^{*}\right\|_{\infty} / H$ is as large as 0.84 . However, the Green-Naghdi equation is a complicated ordinary differential equation whose solutions cannot be found analytically even for a simple short bump.

We conclude that the simple sfKdV is a good asymptotic model in the sense that the relative error is less than $10 \%$ when the bump height is less than one half of the upstream depth and the base of the bump is not longer than twice the bump height. When the bump height is between $50 \%$ and $75 \%$ of the upstream depth and the base of the bump is very long, the Green-Naghdi model, which is a more complex model than the sfKdV, is applicable. When the height of the bump is larger than $75 \%$ of the upstream depth, the free surface will most likely break unless the base of the bump is extremely long.

Acknowledgment. This work was partially supported by the Natural Sciences and Engineering Research Council of Canada.

\section{REFERENCES}

[1] R. M. Miura, The Korteweg-de Vries equation: A survey of results, SIAM Review 18, 412-459 (1976)

[2] A. Patoine and T. Warn, The interaction of long, quasi-stationary baroclinic waves with topography, J. Atmos. Sci. 39, 1018-1025 (1982)

[3] T. R. Akylas, On the excitation of long nonlinear water waves by a moving pressure distribution, J. Fluid Mech. 141, 455-466 (1984)

[4] T. Y. Wu, Generation of upstream-advancing solitons by moving disturbances, J. Fluid Mech. 184, 75-99 (1987)

[5] S. S. P. Shen, Disturbed critical surface waves in a channel of arbitrary cross section, J. Appl. Math. Phys. (ZAMP) 40, 216-229 (1989)

[6] S. S. P. Shen, Forced solitary waves and hydraulic falls in two-layer flows, J. Fluid Mech. 234, 583-612 (1992)

[7] S. J. Lee, G. T. Yates, and T. Y. Wu, Experiments and analyses of upstream-advancing solitary waves generated by moving disturbances, J. Fluid Mech. 199, 569-593 (1989)

[8] A. S. Peters and J. J. Stoker, Solitary waves in liquids having non-constant density, Comm. Pure Appl. Math. 13, 115-164 (1960)

[9] I. Stakgold, Green's Functions and Boundary Value Problems, John Wiley and Sons, Chapter 9, New York, 1979

[10] J. W. Miles, Stationary, transcritical channel flow, J. Fluid Mech. 162, 489-499 (1986)

[11] N. S. Sivakumaran, T. Tinganchali, and R. J. Hoskings, Steady shallow flow over curved beds, J. Fluid Mech. 128, 469-487 (1983) 
[12] P. M. Naghdi and L. Vongsarnpigoon, The downstream flow beyond an obstacle, J. Fluid Mech. 162, 223-236 (1986)

[13] F. Dias and J.-M. Vanden-Broeck, Open channel flows with submerged obstructions, J. Fluid Mech. 206, 155-170 (1989)

[14] J. L. Hammack and H. Segur, The Korteweg-de Vries equation and water waves. Part 2. Comparison with experiments, J. Fluid Mech. 65, 289-314 (1974) 